\title{
Two-dimensional grain boundary sliding and mantle dislocation accommodation in ODS ferritic steel
}

Hiroshi Masuda $^{\mathrm{a}, \mathrm{b}}$, Hirobumi Tobe ${ }^{\mathrm{a}}$, Eiichi Sato ${ }^{\mathrm{a}}$, Yoshito Sugino ${ }^{\mathrm{c}}$, Shigeharu Ukai ${ }^{\mathrm{d}}$

${ }^{a}$ Institute of Space and Astronautical Science, Japan Aerospace Exploration Agency, 3-1-1

Yoshinodai, Chuo, Sagamihara, Kanagawa 252-5210, Japan.

${ }^{\mathrm{b}}$ Department of Materials Engineering, The University of Tokyo, 7-3-1 Hongo, Bunkyo, Tokyo 113-8654, Japan.

${ }^{c}$ Kobelco Research Institute, Inc., 2-3-1 Shinhama, Arai, Takasago, Hyogo 676-8670, Japan.

${ }^{\mathrm{d}}$ Materials Science and Engineering, Faculty of Engineering, Hokkaido University, N13, W-8, Kita-ku, Sapporo, Hokkaido 060-8628, Japan.

Corresponding author: Hiroshi Masuda

Phone number: +81-50-3362-7539

E-mail address: masuda.hiroshi@ac.jaxa.jp

Postal address: 3-1-1 Yoshinodai, Chuo, Sagamihara, Kanagawa 252-5210, Japan

E-mail address

Hiroshi Masuda: masuda.hiroshi@ac.jaxa.jp

Hirobumi Tobe: tobe@isas.jaxa.jp

Eiichi Sato: sato@isas.jaxa.jp

Yoshito Sugino: y-sugino@frontier.hokudai.ac.jp

Shigeharu Ukai: s-ukai@eng.hokudai.ac.jp 


\begin{abstract}
The mechanism governing grain boundary sliding (GBS) accommodated by dislocation and microstructural evolution in regions II/III and III was studied to understand superplasticity. Two-dimensional GBS that occurred during high-temperature shear in oxide dispersion strengthened ferritic steel exhibiting an elongated and aligned grain structure was analyzed using surface markers drawn by focused ion beams. In addition, the accommodating dislocation structure was evaluated by electron back-scattered diffraction and electron channeling contrast imaging. In the initial stage of deformation, GBS triggered dislocation slippage in "mantle" areas near grain boundaries. These mantles tended to appear around GBS-resistant areas such as curved boundaries and grain protrusions. Next, the mantle dislocations generated dislocation walls before forming low-angle boundaries (LABs) along $\{110\}$ crystallographic planes via dynamic recovery at the core/mantle boundaries. Finally, secondary GBS or rigid rotation occurred at the newly formed LABs to compensate for the initial GBS and resulted in continuous dynamic recrystallization. These mantle dislocation activities and substructural evolution mechanisms were graphically modeled and validated by comparison with previous studies.
\end{abstract}

\title{
Keywords
}

superplasticity, continuous dynamic recrystallization, dynamic recovery, electron back-scattered diffraction (EBSD), electron channeling contrast imaging (ECCI) 


\section{Introduction}

Evading the trade-off between material strength and formability presents a major challenge. These properties can become compatible by applying structural superplasticity, a state observed in fine and polycrystalline materials under particular conditions. Materials exhibiting high specific strength but low formability such as titanium or aluminum alloys also achieve extremely high plasticity, making them attractive for many fields such as the aerospace industry [1]. However, the mechanism governing superplasticity remains poorly understood, which precludes the prediction and control of microstructural evolution during superplastic forming.

Recently, Alabort et al. [2] have shown that microstructural evolution depended significantly on strain rates in a superplastic titanium alloy. According to their report, grains became finer via dynamic recrystallization at high strain rates but coarser via grain growth at slow rates. However, the underlying mechanism of these microstructural evolutions remains unclear, which limits the understanding of the fundamental principles of superplasticity.

The relative motion of grains with respect to one another along their boundary, or grain boundary sliding (GBS), and its accommodation have been widely believed to predominantly occur in the superplastic state. This accommodation, during which grains undergo deformation to relieve the GBS-induced stress concentration, is regarded as rate-controlling process of superplasticity. However, the existing mechanisms remain controversial. Ball and Hutchison [3] have suggested a superplasticity model of GBS accommodated by intragranular dislocation activities. Gifkins [4] has suggested that dislocation activities may be limited to narrow "mantle" areas along grain boundaries (core-mantle model). In contrast, Ashby and Verrall [5] have proposed a model of diffusion-accommodated GBS and grain switching. Numerous studies support either of these models [6-9] but there is no widespread 
agreement so far. Therefore, a consensus requires a careful study based on a direct mechanistic observation of superplasticity.

"Floating grains" represent a critical bottleneck during microstructural observations of superplasticity. Superplasticity and GBS have been evaluated by surface observation using line markers $[10,11]$, which leave discontinuous gaps at grain boundaries when GBS occurs. However, some grains escape from the free surface during conventional tensile tests. These so-called "floating grains" do not need to undergo deformation by accommodation in the superplastic state because they do not interact with neighboring grains. They may behave in a manner irrelevant to their bulk counterparts, which may skew observations of superplasticity. Watanabe et al. [12] have detected different texture formations during superplasticity near the surface compared with in the bulk, emphasizing that surface observation may provide misleading results.

To avoid floating grains, Mayo and Nix [13], Rust and Todd [14], and Alabort et al. [15] have conducted shear tests, and deformed specimens within two dimensions because no floating grain would result from a truly two-dimensional deformation. This approach prevented the emergence of new grains from the subsurface at the free surface despite the presence of microscopic floating grains in certain parts. Rust et al. [14] investigated whether these microscopic grains presented any problem through careful experiments and discovered different phenomena at and beneath the surface even in the same grain. In particular, a grain-switching event actually occurred below the surface without appearing on the surface. This difference may be attributed to the absence of interaction between floating grains on the surface.

Even in a macroscopically two-dimensional deformation, out-of-plane grain movements have been unavoidable as long as the grains are equiaxed [13-15]. If an anisotropic microstructure comprising elongated and aligned grains were sheared perpendicularly to the 
longitudinal direction, grain movement would only occur in two dimensions (Fig. 1). This idea is similar to previous attempts by Muto et al. [16], who deformed a bundle of pencils acting as a two-dimensional model aggregate and evaluated their sliding behaviors. Based on this concept, two-dimensional GBS has been achieved and investigated in oxide dispersion strengthened (ODS) ferritic steel exhibiting an elongated and aligned grain structure [17-20]. Although this alloy does not make a superplastic elongation over hundreds of percent but a sigmoidal stress-strain rate relationship and two-dimensional GBS, which have been highly useful for interpreting an initial stage of superplasticity.

In our former work [18], the deformed microstructures were evaluated by electron back-scattered diffraction (EBSD) in conjunction with surface microgrids drawn by focused ion beam (FIB) in regions II (optimum for GBS), II/III (border), and III (dislocation creep), respectively. Significant GBS was detected in regions II and II/III, where the density of geometrically necessary dislocations (GNDs) increased around the sliding boundaries. The GND density increased more significantly in region II/III than in region II, suggesting a larger contribution of dislocation activities in region II/III. On the other hand, non-dislocation mechanism, e.g. diffusion of matter, might result in more contribution in region II.

Local accommodations via dislocation activities have been observed in region II/III [19, 20]. Dislocation slippage accommodated GBS in mantle areas near sliding boundaries only [19], which is consistent with the "core-mantle model" proposed by Gifkins [4], and produced new low-angle boundaries (LABs) cutting grain protrusions [20]. However, details of the dislocation accommodation and microstructural evolution have yet to be studied comprehensively.

In this study, the GBS-triggered mantle dislocation mechanism and its effects on microstructural evolution were unified and a comprehensive model was graphically proposed. Shear tests were conducted to achieve more refined evaluation than ever. Compared with 
conventional tensile tests that generated two GBS modes around $45^{\circ}$ and $135^{\circ}$ with respect to the tensile axis $[18,19]$, shear tests produced only one GBS mode along the shear direction. This one-mode GBS is expected to facilitate a better characterization of GBS networks and analysis of accommodations such as intragranular dislocation activities.

\section{Experimental}

\subsection{Material preparation}

This study rests on the preparation of an anisotropic microstructure consisting of fine and elongated grains for two-dimensional GBS.

The alloy composition of ODS ferritic steel used here is shown in Table 1. Metal and $\mathrm{Y}_{2} \mathrm{O}_{3}$ powders were mechanically alloyed in an argon-gas atmosphere and hot-extruded at $1423 \mathrm{~K}$ into a round bar featuring dispersed nanosized $\mathrm{Y}_{2} \mathrm{O}_{3}$ particles. An almost identical material comprised spherical $\mathrm{Y}_{2} \mathrm{O}_{3}$ particles averaging $5 \mathrm{~nm}$ in diameter and occupying a volume fraction of $0.40 \mathrm{vol} \%$ [21]. The extruded sample was cold-rolled to achieve a reduction of $85 \%$ and annealed at $1423 \mathrm{~K}$ for $4 \mathrm{~h}$ before water quenching to create a recrystallized microstructure. The $\mathrm{Y}_{2} \mathrm{O}_{3}$ particles pinned grain boundaries and prevented their migration. Therefore, the grain sizes and shapes depended on the particle distribution. Figure 2 shows inverse pole figure (IPF) maps obtained from EBSD analyses of an as-annealed sample. The recrystallized grains were largely elongated and aligned along the rolling direction $(\mathrm{RD})$.

\subsection{Specimen preparation}


A schematic representation of a shear test specimen is shown in Fig. 3a. First, the as-annealed sample was cut into a tensile specimen with gauge width of $1.5 \mathrm{~mm}$ and thickness of $0.7 \mathrm{~mm}$. Then, a pair of diagonally symmetrical U-shaped notches with a depth of $0.30 \mathrm{~mm}$, radius of $0.15 \mathrm{~mm}$, and interval of $0.7 \mathrm{~mm}$ was produced by micro-electric discharging.

Figure $3 \mathrm{~b}$ shows a secondary-electron image (SEI) of the RD surface of a shear test specimen with a pair of U-shaped notches, which is expected to cause shear deformation between the notches in a plane tilted by $15^{\circ}$ with respect to the ND plane under tension.

The examined surface (RD plane) was mechanically polished and subsequently electro-polished in a 1:9 perchloric acid/acetic acid mixture at $15 \mathrm{~V}$ for $60 \mathrm{~s}$. Three kinds of markers were drawn by FIB on the polished surface. "Coarse markers" were prepared perpendicularly to the expected shear plane (tilted by $15^{\circ}$ to the ND) with intervals of $20 \mu \mathrm{m}$ (Figs. 3c and d). "Point markers" were generated parallel and perpendicularly to the shear direction at $40 \mu \mathrm{m}$ intervals (Fig. 3d). Mesoscopic shear strains measured from these point markers are shown together with microstructural observations below. "Fine markers" were positioned across grain boundaries at $0.5 \mu \mathrm{m}$ intervals (Fig. 3e).

Surface marker shapes were measured by scanning probe microscopy (SPM) (Fig. $3 f$ and g). Coarse and fine marker depths averaged 120 and $53 \mathrm{~nm}$, respectively.

\subsection{Mechanical testing}

High-temperature shear tests were performed at $1173 \mathrm{~K}$ under vacuum $\left(<10^{-3} \mathrm{~Pa}\right)$ in a hydraulic servo fatigue testing machine coupled with a high-frequency induction heating facility using constant loads, 13, 17, 20, and $26 \mathrm{~N}$. The nominal von Mises stress applied in the deformed areas were calculated by a finite element method supposing a two-dimensional 
elastic model, amounting to $63 \pm 11,49 \pm 8.1,41 \pm 6.9$, and $32 \pm 5.3 \mathrm{MPa}$, respectively. The tests were conducted to fracture which initiated from the notches in all the specimens. The fractured specimens were cooled in the chamber. The average cooling rate was ca. $3 \mathrm{~K} / \mathrm{s}$ from $1173 \mathrm{~K}$ (testing temperature) to $973 \mathrm{~K}$, above which Leng et al. [22] have reported that static recovery got significant in the similar material. Shear strains were measured using the displacement of the point markers after deformation and divided by the testing time to derive the strain rates.

\subsection{Microstructural characterization}

GBS and its accommodating deformations were evaluated by field-emission scanning electron microscopy (FE-SEM) and confocal laser microscopy (CLM). Microstructural evolution was examined in several replicates before and after deformation using EBSD and electron channeling contrast imaging (ECCI). Post-deformation analyses required surface oxide layer removal, which was conducted by $\mathrm{Ar}^{+}$-ion polishing at $3.0 \mathrm{kV}$ for $30 \mathrm{~min}$ followed by electro-polishing for $5 \mathrm{~s}$ for EBSD and ECCI, respectively, to reach a total polishing depth of about $1 \mu \mathrm{m}$.

Specifically, EBSD analyses were performed at an acceleration voltage of $15 \mathrm{kV}$ with step sizes of $0.2 \mu \mathrm{m}$. In the rebuilt maps, low- $\left(4^{\circ}-15^{\circ}\right)$ and high-angle boundaries $\left(>15^{\circ}\right)$ appeared in blue and black, respectively. Kernel average misorientation (KAM) values were also mapped to visualize the GND density distribution. Unrecrystallized grains that initially exhibited high GND density were excluded from the KAM maps after deformation. ECCI analyses were conducted at an acceleration voltage of $30 \mathrm{kV}$ to observe the grain boundaries and dislocation structures in detail. 


\section{Results}

\subsection{Mechanical behavior}

All the specimens fractured from the tips of the U-shaped notches. Figures $4 \mathrm{a}$ and $\mathrm{b}$ show the shear strain distributions measured from the point markers after deformations at 26 and $20 \mathrm{~N}$. The deformations were not highly homogeneous but confirmed to be a shear type as expected. The average shear strain rates, $\dot{\gamma}$, were calculated from the average strains to be divided by the testing times.

Figure 4d shows CLM profiles of the surface altitude along the dashed arrows in Figs. 4a and $\mathrm{b}$. These profiles show only small changes in altitude within a sub-micron range, which imply the minor contribution of out-of-plane shears by floating grains compared to the in-plane deformations.

Figure 5 shows logarithmic plots of the nominal stress as a function of the average strain rate at $1173 \mathrm{~K}$, exhibiting a typical translation between regions II and III. In these plots, the slopes represent the strain rate sensitivity index $(m)$ values. The $m$ significantly changed around $4 \times 10^{-5} \mathrm{~s}^{-1}$, which corresponds to the border between regions II and III (region II/III). The $m$ reached 0.3 in region II, closely matching the previous result obtained in tension at the same temperature [18].

\subsection{Microstructural observations}

\subsubsection{Grain boundary sliding behaviors}

Figure 6 shows SEIs after deformations in regions III (1173 K, 26 N, 12\%), II/III (1173 K, 
$17 \mathrm{~N}, 10 \%)$, and II (1173 K, $13 \mathrm{~N}, 5 \%)$, respectively. The deformed microstructures can be characterized as follows.

(i) Coarse markers displayed micron-scale gaps at all the strain rates (Figs. 6a, c, and e), confirming the occurrence of GBS. On the other hand, out-of-plane GBS was rather negligible according to Fig. 4c. These results mean that GBS behaviors were almost two-dimensional.

(ii) Magnified images show the curvatures of the coarse markers near the sliding boundaries, i.e. within mantle areas, in regions III (Fig. 6b) and II/III (Fig. 6d). These curvatures resulted from mantle dislocation slippage.

(iii) A similar curvature was also observed in grain centers in region III as indicated by the white arrow in Fig. 6a. Such dislocation activities in core areas will be reported in detail in our future publication.

The above results suggest that dislocation activities were the operative accommodations of GBS in regions II/III and III. Characteristics of the dislocation accommodations depended on the deformation conditions. Specifically, dislocation activities were limited within mantles in region II/III but also observed in cores in region III. Significant GBS was also detected in region III, where small amount of GBS had previously been identified [18].

This study will mainly evaluate the mantle dislocation activities observed in regions II/III and III. The following sections assess a series of characteristic microstructures in the targeted regions. These microstructures represent different stages of the mantle dislocation mechanism accommodating GBS.

3.2.2. Dislocation slippage in mantles

Figure 7 shows SEIs of fine markers deformed in region II/III (1173 K, $17 \mathrm{~N})$, 
highlighting the grain boundaries in yellow, intragranular-deformed areas in orange, and original markers in red, respectively. In all of these photographs, several characteristic relationships can be confirmed between GBS and the accommodating intragranular deformation:

(i) GBS and the accommodating intragranular deformation could occur simultaneously.

(ii) The fraction of GBS increased at flat boundaries while that of intragranular deformation increased in grain protrusions or around curved boundaries.

(iii) Intragranular deformation occurred to fill up curved boundaries.

These characteristics suggest that mantle regions exhibiting intragranular deformations appeared in GBS-resistant areas, especially in grain protrusions and around curved boundaries. These intragranular deformations are indicative of dislocation slippage in mantle regions.

\subsubsection{Dislocation accumulation in mantles}

Figure 8 shows EBSD, SEI, and ECCI results obtained in regions III (1173 K, 26 N, 9.1\%) and II/III (1173 K, $20 \mathrm{~N}, 11 \%)$, respectively.

Figures $8 \mathrm{a}$ and $\mathrm{d}$ show initial IPF maps of the recrystallized microstructures. The SEIs acquired after deformation revealed gaps and curvatures of coarse markers (Figs. 8b and e), consistent with GBS and near-boundary deformation, respectively. The corresponding KAM maps (Figs. 8c and f) demonstrate that the GND density increases significantly around the sliding boundaries. In general, these GNDs fill up grain protrusions that might act as obstacles to GBS.

Figures $8 \mathrm{~g}$ and h show ECC images obtained from the marked area in the SEI (rectangle, Fig 8e). A dislocation array was observed in the grain protrusion. The grain (bottom, Figs. $8 \mathrm{~g}$ 
and h) was divided into two different regions: a peripheral "mantle" accompanied by the dislocation structure and a central "core" presenting negligible dislocations.

Figure $8 \mathrm{i}$ shows misorientation profiles before and after deformation derived from the EBSD data (dashed arrows, Figs. 8d and f). A gradual lattice rotation of $5^{\circ}$ is observed within $0.6 \mu \mathrm{m}$ from the grain boundary, indicative of the existence of GNDs. This narrow area exhibits a consistent width with respect to the mantle detected by ECCI (Fig. 8g).

Overall, these results suggest that GBS triggered intragranular deformation, such as dislocation slippage, and enhanced GND density in mantle areas, which emerged in GBS-resistant areas.

\subsubsection{Low-angle boundary formation}

In this section, substructural evolutions following the dislocation slippage and accumulation in the mantles were examined. Figure 9 shows the EBSD, SEI, and ECCI results in regions III (1173 K, $26 \mathrm{~N}, 9.2 \%)$ and II/III $(1173 \mathrm{~K}, 20 \mathrm{~N}, 7.5 \%)$ obtained in different areas from Section 3.2.3.

Figures 9a and d show the initial IPF maps of the recrystallized microstructures. After deformation, the SEIs present gaps characteristic of GBS across the coarse markers (Figs. 9b and e). Grain protrusions that behaved as obstacles to GBS are also detected (arrows, Figs. 9b and e), as described above (Section 3.2.3). These protrusions display a significant increase in GND density in KAM maps (Figs. 9c and f).

The dislocation density increased similarly to that shown above (Section 3.2.3). In addition, the KAM maps after deformation display newly generated LABs (blue lines, Figs. 9c and f). These LABs present two remarkable characteristics. They formed (i) to cut grain protrusions from their original grains and (ii) along $\{110\}$ crystallographic planes, which are 
the densest planes in a bcc metal.

Figure 9g shows an ECC image in region II/III (rectangle, Fig. 9e), where the stable LAB is clearly distinguished from the dislocation array.

Figure $9 \mathrm{~h}$ shows misorientation profiles before and after deformation (dashed arrows, Figs. 9d and f). The profile obtained after deformation confirms the formation of the new LAB (ca. $8^{\circ}$ ) and small lattice rotation typical of GNDs between this LAB and the original boundary.

Figure 10 also shows misorientation profiles across newly formed substructures around mantle areas randomly chosen after deformation in regions III $(1173 \mathrm{~K}, 26 \mathrm{~N})$ and II/III $(1173$ $\mathrm{K}, 20 \mathrm{~N}$ ), respectively. These profiles indicate the substructures including LABs (LAB type) in solid lines and those without an LAB (dislocation wall type) in dotted lines. The LABs can be confirmed only in the substructures with misorientations larger than $5^{\circ}$ or so. These results suggest that LABs resulted from dynamic recovery when the misorientations of mantle dislocation walls reached ca. $5^{\circ}$.

The above results provide clear evidence for microstructural evolution. Mantle dislocation activities resulted in the formation of new LABs via dynamic recovery. This dynamic recovery, which involved the rearrangement of accumulated dislocations, occurred in a geometrical position cutting grain protrusions from their original grains, which may be characteristic of mantle dislocation accommodation, as discussed in Section 4.1.

\subsubsection{Rigid rotation at a new low-angle boundary}

In this section, a contribution of newly formed LABs to subsequent deformation via local rigid rotation was investigated. Figure 11 shows EBSD, SEI, and ECCI analyses in region III $(1173 \mathrm{~K}, 26 \mathrm{~N}, 18 \%)$ obtained in different areas from Section 3.2.3 and 3.2.4. 
Figures 11a and b show IPF maps of the same area before and after deformation, respectively. Interestingly, a new grain boundary formed in the central grain, cutting its protrusion during deformation. Gaps detected in coarse markers confirmed the occurrence of GBS (Fig. 11d). These observations suggest that the newly formed boundary resulted from the cutting of the grain protrusion.

Figure 11c shows misorientation profiles across the protrusion before and after deformation (dashed arrows, Figs. 11a and b). The newly formed boundary exhibited a rotation angle of $17^{\circ}$, closely matching the rigid rotation angle observed in a grain protrusion (Fig. 11e). This suggests that this boundary (Figs. 11b and c) resulted from a LAB formation followed by an angle increase via rigid rotation. As a result, the protrusion corresponds to a mantle area, consistent with the gradual lattice rotation confirmed in the orientation profile (Fig. 11c) and the dislocation structure observed by ECCI (Fig. 11f).

Overall, dislocation activities in the mantle led to a new LAB that cut the grain protrusion, where local rigid rotation produced the necessary shear deformation to compensate for GBS strain.

\section{Discussion}

4.1 Models for mantle dislocation activities and cutting mechanism

Microstructural observations in Section 3.2 have given a series of images of the GBS-triggered mantle dislocation mechanism and microstructural evolution in regions II/III and III (Fig. 12). In the figure, dislocations are illustrated in an edge style, but not necessarily.

In the initial stage of deformation, dislocations play a role in the relaxation of GBS-derived stress concentration by nucleating and slipping in mantle areas near grain 
boundaries to compensate for GBS strain (Fig. 12b). Mantle areas dominate GBS-resistant sites, especially in grain protrusions or around curved boundaries.

These dislocation activities cause substructural evolutions. Mantle dislocations having a motion component toward core areas are trapped at core/mantle boundaries because these dislocations are prevented from moving freely in core areas with low stress (Fig. 12c). These trapped and accumulated dislocations around core/mantle boundaries are transformed into dislocation walls before generating LABs along $\{110\}$ crystallographic planes via dynamic recovery (Fig. 12d). Consequently, LABs tend to appear in geometrical positions cutting grain protrusions from their original grains.

According to Taylor et al. [23], dislocation walls and cell boundaries tend to form along the densest crystallographic planes in fcc alloys, such as $\{111\}$ planes, during continuous dynamic recrystallization, in agreement with the current observations. Possibly, the increasing dislocation density around core/mantle boundaries accommodating GBS acted as a driving force for local dynamic recovery and triggered the formation of LABs along $\{110\}$ planes in the present case.

The cutting mechanism leaves new small subgrains around original boundaries, which result in grain refinement as continuous dynamic recrystallization. According to micrographs shown by Alabort et al. [15], the subgrain formation apparently started around an original curved boundary during superplasticity in region II/III. Soer et al. [24] have also reported that subgrains initially formed around mantle areas during continuous dynamic recrystallization in a superplastic $\mathrm{Al}-\mathrm{Mg}$ alloy. Both of their findings $[15,24]$ can be well explained by the cutting mechanism.

Finally, newly formed LABs produce the succeeding deformations, such as secondary GBS or rigid rotation, and compensate for GBS strain (Fig. 12e). Here, a local rigid rotation was confirmed to produce a shear strain compensating for GBS (Fig. 11). 
Review the study by Geckinli et al. [25], and evidence of a secondary GBS at a new LAB resulting from the cutting mechanism can be found. A pair of SEM micrographs (Fig. 13) tracking the movement of two grains termed "g" and " $h$ " during superplasticity in $\mathrm{Pb}-\mathrm{Sn}$ eutectic was used to measure the rigid rotation of each grain. However, a detailed review of these micrographs provides a new insight that GBS did not occur at the original grain boundary but at a core/mantle boundary cutting a protrusion of grain " $\mathrm{h}$ ", as additionally illustrated. These micrographs clearly provide strong evidence of a sequential process of GBS at a new LAB via the cutting mechanism.

This model for the mantle dislocation activities and cutting mechanism accommodating GBS in regions II/III and III (Fig. 12) provides a new insight into superplasticity and aids the reinterpretation of previous studies.

4.2. Verification of the mechanism by reviewing past studies

Earlier studies discussing the accommodating mechanism of GBS were reviewed and reinterpreted to verify the present mechanism. This verification rested on the (i) validity of the mantle dislocation accommodation itself as the mechanism governing superplasticity and (ii) consistency of the deformation conditions of regions II/III and III. In this section, previous studies by Ball and Hutchison [3], Matsuki et al. [26], and Xun and Mohamed [27], who reported the dislocation structures directly observed after superplasticity and proposed dislocation-accommodated GBS as the dominant mechanism for superplasticity, are reviewed.

Ball and Hutchison [3] observed dislocation structures in $\mathrm{Zn}-\mathrm{Al}$ eutectoid deformed in region II/III but not region II. In particular, they identified high-density dislocations around the grain protrusion in an Al-rich grain, and some dislocations formed a cell structure, well 
consistent with this study.

Matsuki et al. [26] studied a single-phase aluminum alloy by deforming it under conditions encompassing regions I to III and analyzing the accommodating mechanisms using surface markers and transmission electron microscopy (TEM). The curvature of the surface markers and dislocations observed by TEM were only reported for higher strain rate conditions than region II/III. Moreover, the observed dislocation arrays were limited to narrow ranges (about $2 \mu \mathrm{m}$ ) from the grain boundaries present in region II/III $(0.2<m<0.4)$ while dislocation structures were elongated into grain centers in region III $(m<0.2)$ according to TEM. These results agree with the present study, in which mantle dislocations are confirmed in regions II/III and III while the transgranular deformation of core areas only occurred in region III (Fig. 6a).

These previous studies support the present mechanism of mantle dislocation accommodation. In addition, they clarify that dislocation structures after superplasticity are commonly observed in higher strain rate conditions than region II/III.

According to Xun and Mohamed [27], however, dislocation structures formed within grain centers (core areas) are presented in regions I and II in $\mathrm{Zn}-\mathrm{Al}$ eutectoid containing nano-scale dispersion particles. Their observations appear to contradict the present results and other studies reviewed above $[3,26]$ because they have shown a consistent tendency that dislocations become more suppressed under lower strain rate conditions. It should be noted, however, that the dislocations observed in regions I and II [27] have shown two differences from their counterparts detected in regions II/III and III [3, 26]. Specifically, (i) the dislocations adopted a parallel alignment below region II instead of a complicated substructural evolution above region II/III, and (ii) dislocations below region II cannot be maintained without dispersion particles but those above region II/III are preserved alone after deformation. These differences between dislocation characteristics imply that another 
dislocation accommodation operates in regions I and II. Xun and Mohamed concluded that the mechanism was the Ball-Hutchison type [3], which is accommodated by transgranular slippage and the pile-up of dislocations.

In contrast, the dislocation-accommodated mechanism below region II remains uncertain. Rust and Todd [14] have proposed another mechanism for region II. They observed a local mass flow around sliding boundaries using FIB-drawn submicron grids and concluded that Spingarn and Nix-type diffusion was the dominant accommodation in region II [7]. Grain growth has been also detected below region II [2, 28-30] and the true deformation mechanism needs to facilitate this microstructural evolution. The mechanisms below region II are expected to be revealed using the present two-dimensional analysis in a future publication.

\section{Conclusions}

Two-dimensional GBS and dislocation accommodations were observed in ODS ferritic steel which is composed of anisotropic grains elongated and aligned in one direction. This alloy did not exhibit a superplastic elongation but its two-dimensional GBS phenomena were useful for constructing the deformation mechanism and the microstructural evolution processes in regions II/III and III as below:

1. GBS was accommodated by dislocation activities, such as slippage and substructural evolution, which were limited within mantle areas near the sliding boundaries.

2. The mantles significantly appeared in GBS-resistant areas, such as near curved boundaries or in grain protrusions.

3. Dislocations slipping in mantles were trapped at core/mantle boundaries and promoted dynamic recovery, which produced LABs along $\{110\}$ crystallographic planes near 
core/mantle boundaries in geometrical positions cutting grain protrusions from their original grains.

4. Newly formed LABs produced subsequent deformations, such as secondary GBS or rigid rotation, to compensate for GBS strain.

5. These processes may correspond to an initial stage of continuous dynamic recrystallization resulting in grain refinement.

\section{Acknowledgement}

This work was supported by Grant-in-Aid for JSPS Fellows [No. 16J04854], Nanotechnology Platform of MEXT, Japan [No. 12024046], and JST under Collaborative

Research Based on Industrial Demand, "Heterogeneous Structure Control: Towards Innovative Development of Metallic Structural Materials". The authors acknowledge Shimadzu Corporation for providing the SPM facility. The authors appreciate the English language review by Enago (www.enago.jp).

\section{References}

[1] E. Sato, S. Sawai, K. Uesugi, T. Takami, K. Furukawa, M. Kamada, M. Kondo, Superplastic titanium tanks for propulsion system of satellites, Mater. Sci. Forum, 551-552 (2007) 43-48.

[2] E. Alabort, D. Putman, R.C. Reed, Superplasticity in Ti-6Al-4V: characterization, modelling and applications, Acta Mater. 95 (2015) 428-442.

[3] A. Ball, M.M. Hutchison, Superplasticity in the aluminium-zinc eutectoid, Met. Sci. 3 (1969) 1-7. 
[4] R.C. Gifkins, Grain-boundary sliding and its accommodation during creep and superplasticity, Metall. Trans. A, 7 (1976) 1225-1232.

[5] M.F. Ashby, R.A. Verrall, Diffusion-accommodated flow and superplasticity, Acta Metall. 21 (1973) 149-163.

[6] A.K. Mukherjee, The rate controlling mechanism in superplasticity, Mat. Sci. Eng. 8 (1971) 83-89.

[7] J.R. Spingarn, W.D. Nix, Diffusional creep and diffusionally accommodated grain rearrangement, Acta Metall. 26 (1978) 1389-1398.

[8] P.M. Hazzledine, J.H. Schneibel, Theory of Coble creep for irregular grain structures, Acta Metall. Mater. 41 (1993) 1253-1262.

[9] T.G. Langdon, A unified approach to grain boundary sliding in creep and superplasticity, Acta Metall. Mater. 42 (1994) 2437-2443.

[10] T.H. Alden, The origin of superplasticity in the Sn-5\%Bi alloy, Acta Metall. 15 (1967) 469-480.

[11] R.B. Vastava, T.G. Langdon, An investigation of intercrystalline and interphase boundary sliding in the superplastic Pb-62\% Sn eutectic, Acta Metall. 27 (1979) 251-257.

[12] H. Watanabe, K. Kurimoto, T. Uesugi, Y. Takigawa, K. Higashi, Accommodation mechanisms for grain boundary sliding as inferred from texture evolution during superplastic deformation, Phil. Mag. 93 (2013) 2913-2931.

[13] M.J. Mayo, W.D. Nix, Direct observation of superplastic flow mechanism in torsion, Acta Metall. 37 (1989) 1121-1134.

[14] M.A. Rust, R.I. Todd, Surface studies of Region II superplasticity of AA5083 in shear: confirmation of diffusion creep, grain neighbor switching and absence of dislocation activity, Acta Mater. 59 (2011) 5159-5170.

[15] E. Alabort, P. Kontis, D. Barba, K. Dragnevski, R.C. Reed, On the mechanism of 
superplasticity in Ti-6Al-4V, Acta Mater., 105 (2016) 449-463.

[16] H. Muto, M. Sakai, The large-scale deformation of polycrystalline aggregates: cooperative grain-boundary sliding, Acta Mater. 48 (2000) 4161-4167.

[17] S. Taniguchi, N. Kawai, E. Sato, Two-dimensional observation of grain movements in elongated and aligned grain structure during high temperature deformation, Mater. Sci. Forum, 735 (2012) 79-83,.

[18] H. Masuda, S. Taniguchi, E. Sato, Y. Sugino, S. Ukai, Two-dimensional observation of grain boundary sliding of ODS ferritic steel in high temperature tension, Mater. Trans. 55 (2014) 1599-1605.

[19] H. Masuda, H. Tobe, E. Sato, Y. Sugino, S. Ukai, Mantle region accommodating two-dimensional grain boundary sliding in ODS ferritic steel, Philos. Mag. Lett. 95 (2015) $359-366$.

[20] H. Masuda, H. Tobe, E. Sato, Y. Sugino, S. Ukai, Two-dimensional observation of the core-mantle model for superplastic flow in an ODS ferritic steel, Mater. Sci. Forum, 838-839 (2016) 100-105.

[21] P. Dou, A. Kimura, R. Kasada, T. Okuda, M. Inoue, S. Ukai, S. Ohnuki, T. Fujisawa, F. Abe, TEM and HRTEM study of oxide particles in an Al-alloyed High-Cr oxide dispersion strengthened steel with Zr addition, J. Nucl. Mater. 444 (2014) 441-453.

[22] B. Leng, S. Ukai, T. Narita, Y. Sugino, Q. Tang, N. Oono, S. Hayashi, F. Wan, S. Ohtsuka, T. Kaito, Effects of two-step cold rolling on recrystallization behaviors in ODS ferritic steel, Mater. Trans. 53 (2012) 652-657.

[23] A.S. Taylor, P. Cizek, P.D. Hodgson, Orientation dependence of the substructure characteristics in a $\mathrm{Ni}-30 \mathrm{Fe}$ austenitic model alloy deformed in hot plane strain compression, Acta Mater., 60 (2012) 1548-1569.

[24] W.A. Soer, A.R. Chezan, J.Th.M. De Hosson, Deformation and reconstruction 
mechanisms in coarse-grained superplastic Al-Mg alloys, Acta Mater. 54 (2006) 3827-3833.

[25] A.E. Geckinli, C.R. Barrett, Superplastic deformation of the Pb-Sn eutectic, J. Mater. Sci. 11 (1976) 510-521.

[26] K. Matsuki, Y. Ueno, M. Yamada, The role of grain boundary sliding in the superplastic deformation of Al-Zn-Mg alloy, J. Japan Inst. Metals, 38 (1974) 219-226.

[27] Y. Xun, F.A. Mohamed, Superplastic behavior of $\mathrm{Zn}-22 \% \mathrm{Al}$ containing nano-scale dispersion particles, Acta Mater. 52 (2004) 4401-4412.

[28] F.A. Mohamed, M.M.I. Ahmed, T.G. Langdon, Factors influencing ductility in the superplastic Zn-22 Pct Al Eutectoid, Metall. Trans. 8A (1977) 933-938.

[29] A.K. Ghosh, C.H. Hamilton, Mechanical behavior and hardening characteristics of a superplastic Ti-6Al-4V alloy, Metall. Trans. 10A (1979) 699-706.

[30] E. Sato, K. Kuribayashi, Superplasticity and deformation induced grain growth, ISIJ Int. 33 (1993) 825-832. 


\section{Caption list}

Fig. 1 Anisotropic microstructure composed of elongated and aligned grains for two-dimensional GBS.

Fig. 2 Initial IPF maps in the (a) ND, (b) TD, and (c) RD planes of recrystallized microstructures.

Fig. 3 (a) Shear test specimen, (b) SEI image of the RD surface of a shear specimen, BSE images of (c) the specimen, (d) coarse and point markers, and (e) fine markers. SPM images of (f) coarse and (g) fine markers.

Fig. 4 Shear strain distributions and average strain rates in (a) region III $(1173 \mathrm{~K}, 26 \mathrm{~N})$ and (b) region II/III (1173 K, $20 \mathrm{~N})$. (c) CLM profiles of surface altitude along the dashed lines in (a) and (b).

Fig. 5 Nominal stress (von Mises) as a function of average strain rates at $1173 \mathrm{~K}$.

Fig. 6 SEIs after deformations in (a) (b) region III (1173 K, 26 N, 12\%), (c) (d) region II/III $(1173 \mathrm{~K}, 20 \mathrm{~N}, 10 \%)$, and (e) (f) region II (1173 K, $13 \mathrm{~N}, 5 \%)$. (b) and (d) are magnified images of the areas marked by rectangles in (a) and (c), respectively.

Fig. 7 SEIs after deformation in region II/III $(1173 \mathrm{~K}, 17 \mathrm{~N})$ to mesoscopic shear strains of (a), (b) 7.6\%, and (c) 8.6\%. Yellow lines, orange areas and red dotted lines represent grain boundaries, intragranular deformed areas and original markers, respectively. 
Fig. 8 (a) (d) Initial IPF maps, (b) SEI, and (c) KAM map of region III (1173 K, 26 N, 9.1\%), (e) SEI, (f) KAM map, and (g) (h) ECC images for magnified areas of region II/III (1173 K, $20 \mathrm{~N}, 11 \%$ ), and (i) misorientation profiles along the dashed arrow in (d) and (f).

Fig. 9 (a) (d) Initial IPF maps, (b) SEI, and (c) KAM map of region III (1173 K, 26 N, 9.2\%), (e) SEI, (f) KAM map, and (g) ECC images for magnified areas of region II/III (1173 K, 20 $\mathrm{N}, 7.5 \%$ ), and (h) misorientation profiles along the dashed arrow in (d) and (f).

Fig. 10 Misorientation profiles across newly formed substructures around mantle areas randomly chosen after deformations in (a) region III $(1173 \mathrm{~K}, 26 \mathrm{~N})$ and $(\mathrm{b})$ region II/III (1173 K, 20 N). Profiles including LABs (LAB type) and those without an LAB (dislocation wall type) are indicated in dotted and solid lines, respectively.

Fig. 11 IPF maps (a) before and (b) after deformation in region III (1173 K, 26 N, 18\%), (c) misorientation profiles along the dashed arrows in (a) and (b), (d) (e) SEIs of the deformed area, and (f) ECC image of a magnified area.

Fig. 12 Proposed mantle dislocation mechanism accommodating GBS: (a) initial state, (b) dislocation slippage in a mantle accommodating GBS, (c) dislocations trapped at a core/mantle boundary, (d) dynamic recovery of the dislocation structure into an LAB along $\{110\}$ crystallographic planes, and (e) subsequent deformation, such as GBS or grain rotation, compensating for GBS strain. 
Fig. 13 SEM photographs during superplastic flow of $\mathrm{Pb}-\mathrm{Sn}$ eutectic at $298 \mathrm{~K}$ at an initial strain rate of $5 \times 10^{-4} \mathrm{~s}^{-1}$ [24] with additional illustrations of deformation by the present authors. Elongation: (a) 13.7\% and (b) 168\% (c) 1976 Springer).

Table 1 Chemical composition of ODS ferritic steel. 


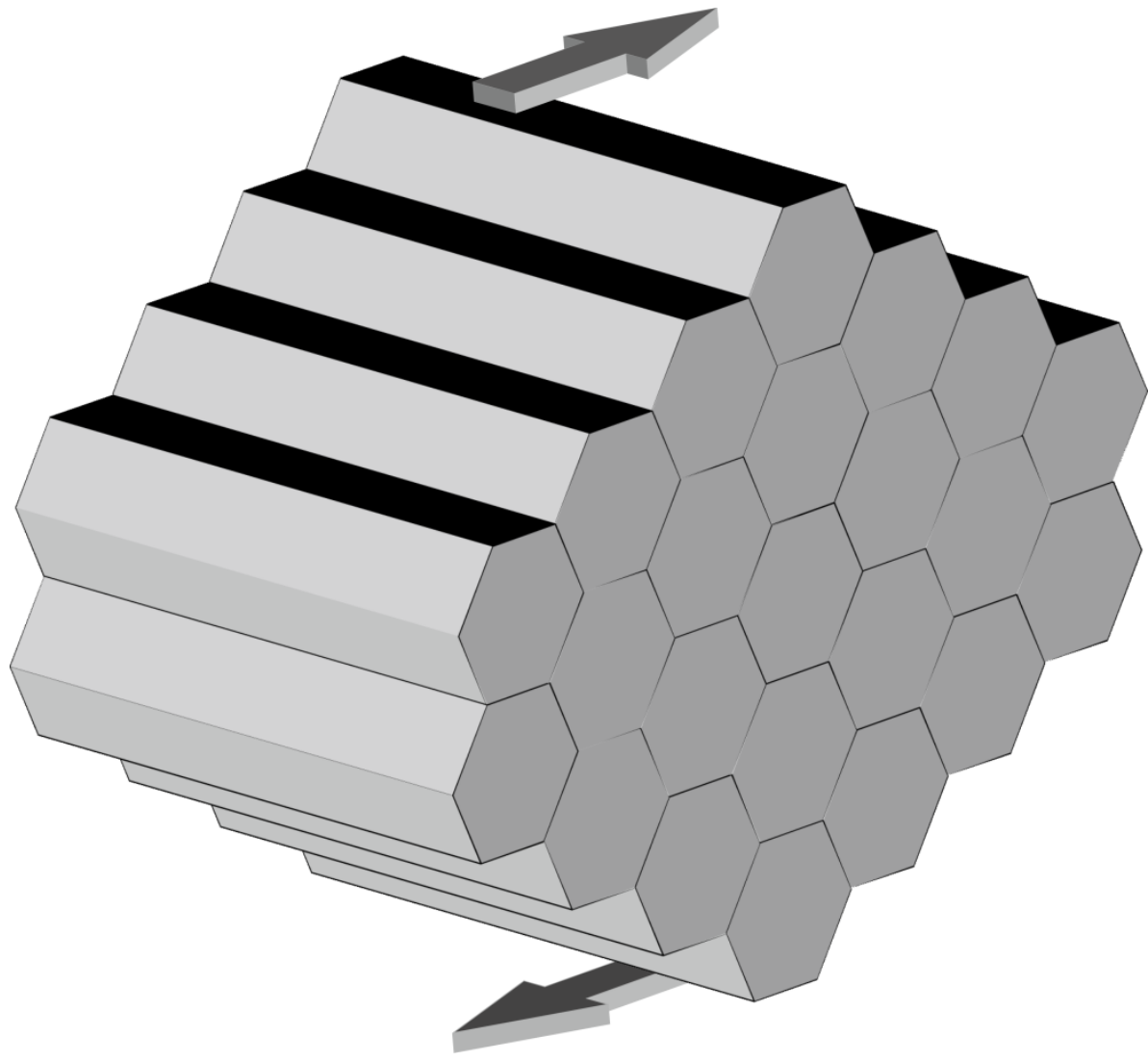

Fig. 1 Anisotropic microstructure composed of elongated and aligned grains for twodimensional GBS. 

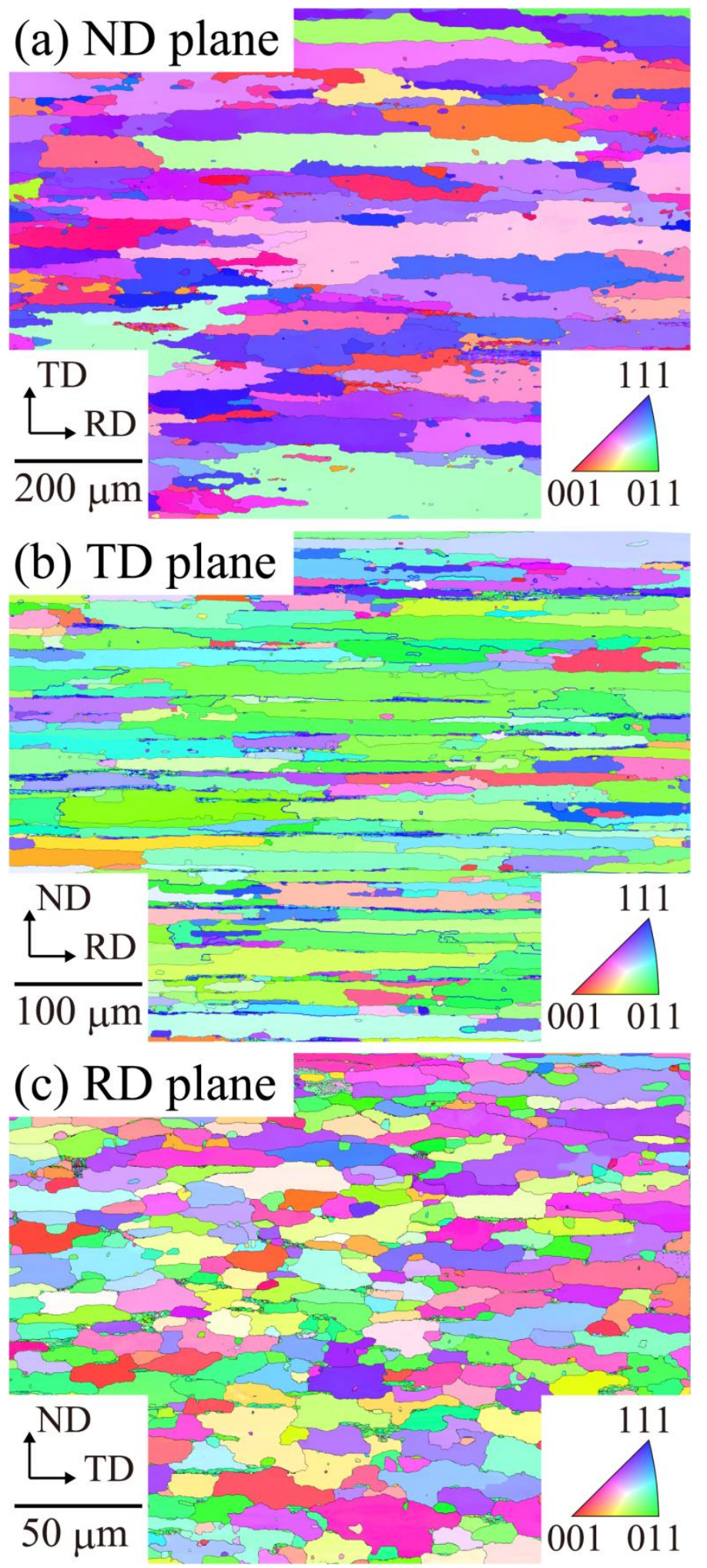

Fig. 2 Initial IPF maps in the (a) ND, (b) TD, and (c) RD planes of recrystallized microstructures. 

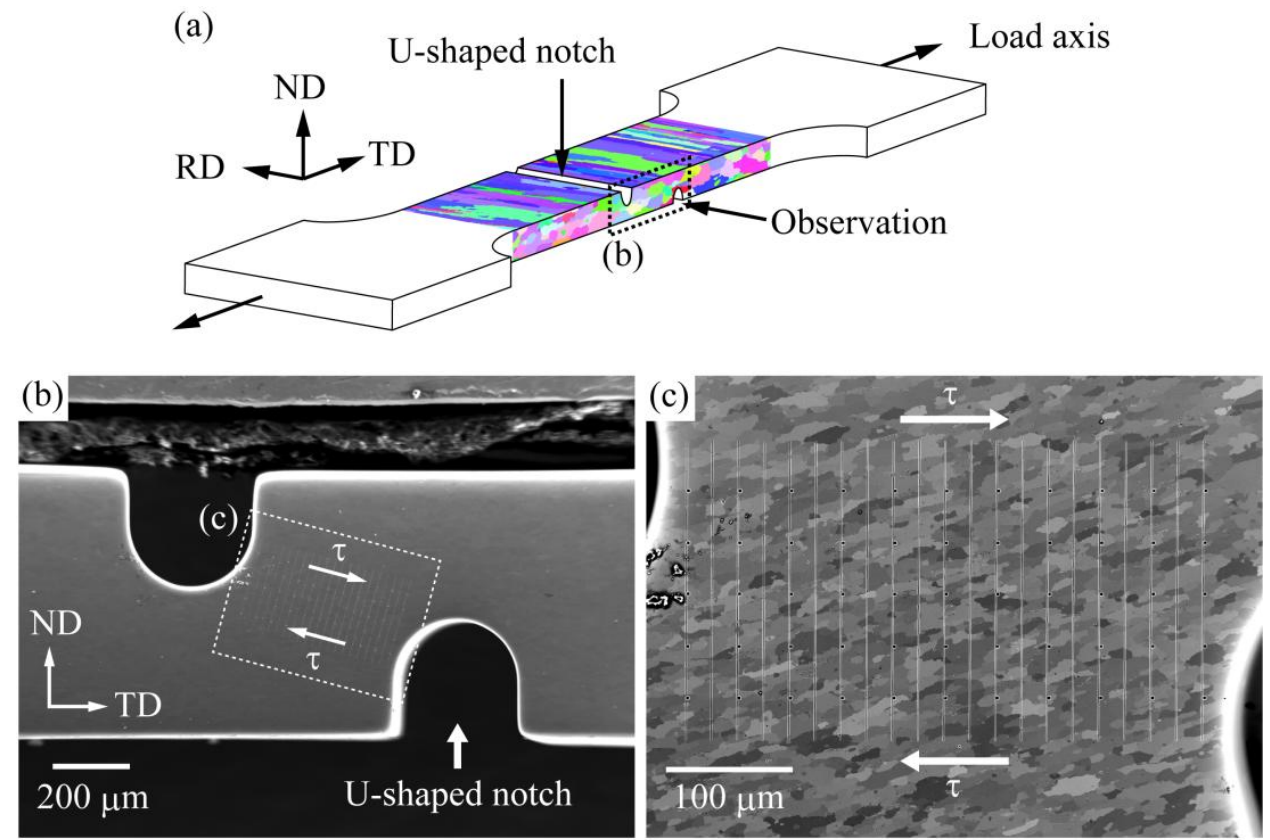

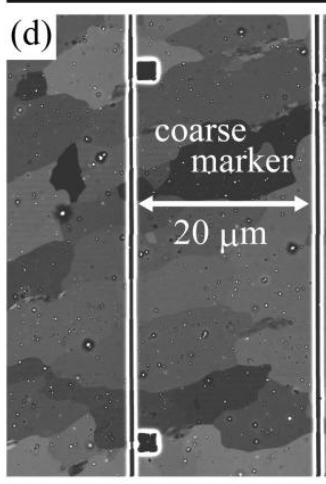

(f)

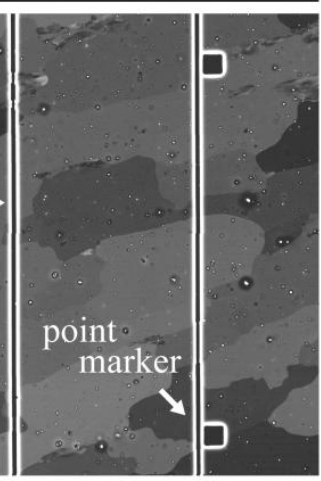

(g)
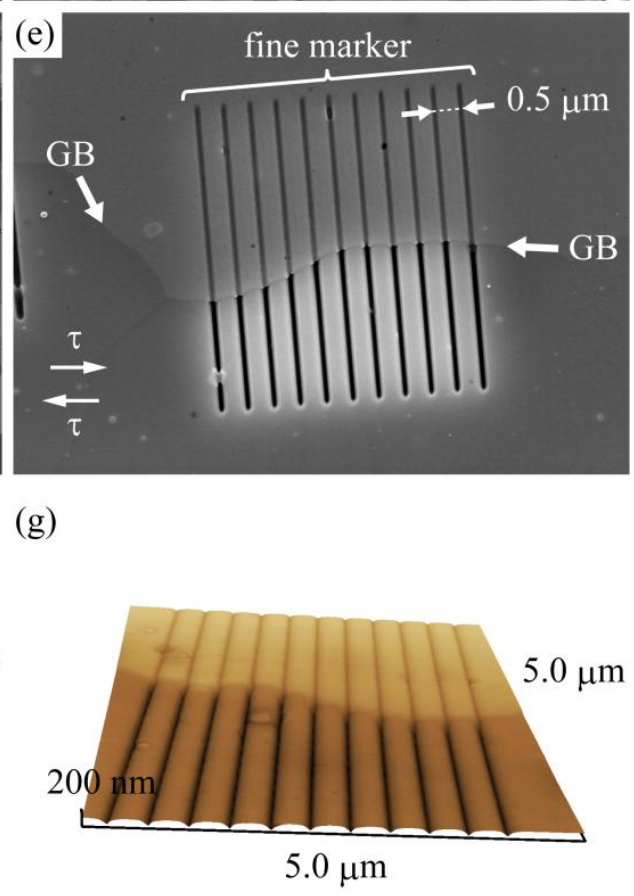

Fig. 3 (a) Shear test specimen, (b) SEI image of the RD surface of a shear specimen, BSE images of (c) the specimen, (d) coarse and point markers, and (e) fine markers. SPM images of (f) coarse and (g) fine markers. 

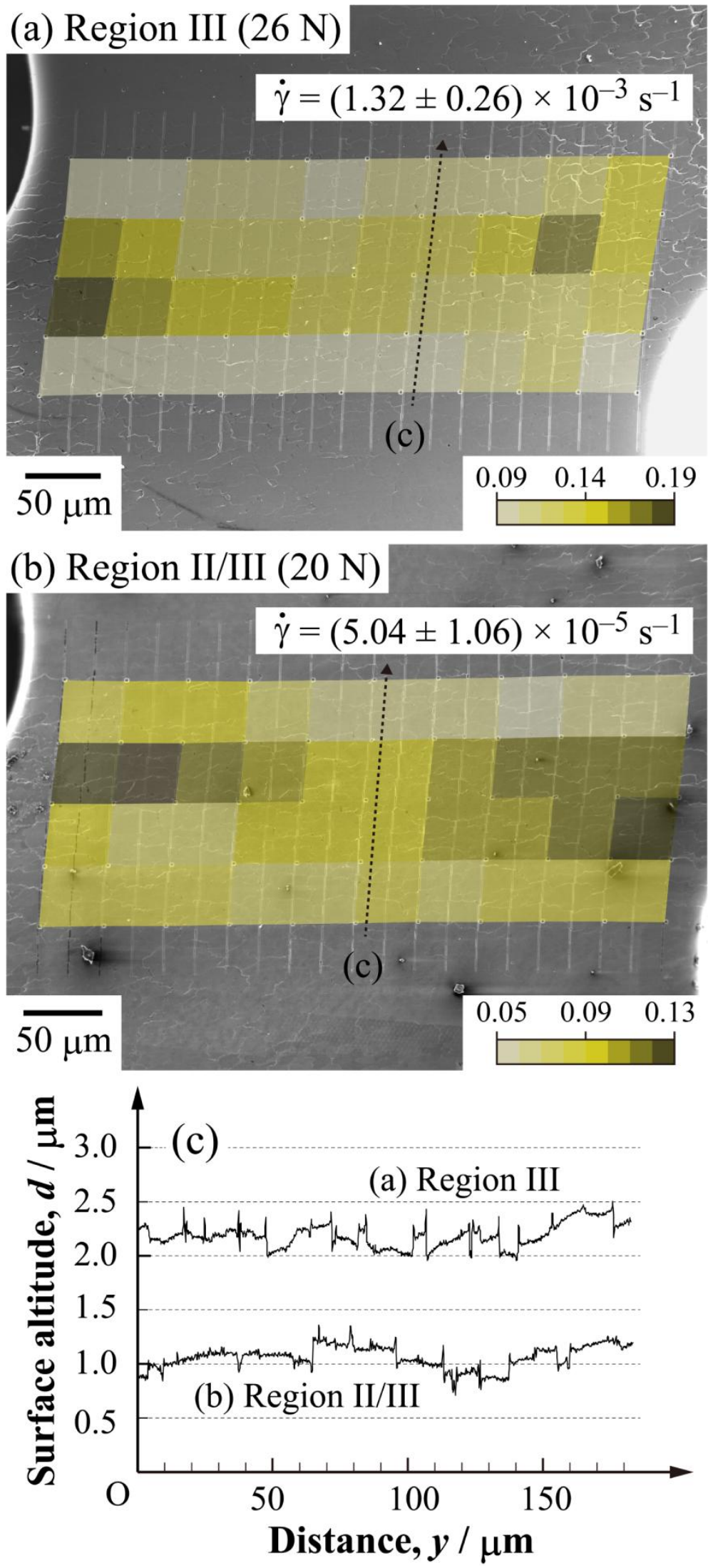

Fig. 4 Shear strain distributions and average strain rates in (a) region III (1173 K, $26 \mathrm{~N})$ and (b) region II/III (1173 K, $20 \mathrm{~N})$. (c) CLM profiles of surface altitude along the dashed lines in (a) and (b). 


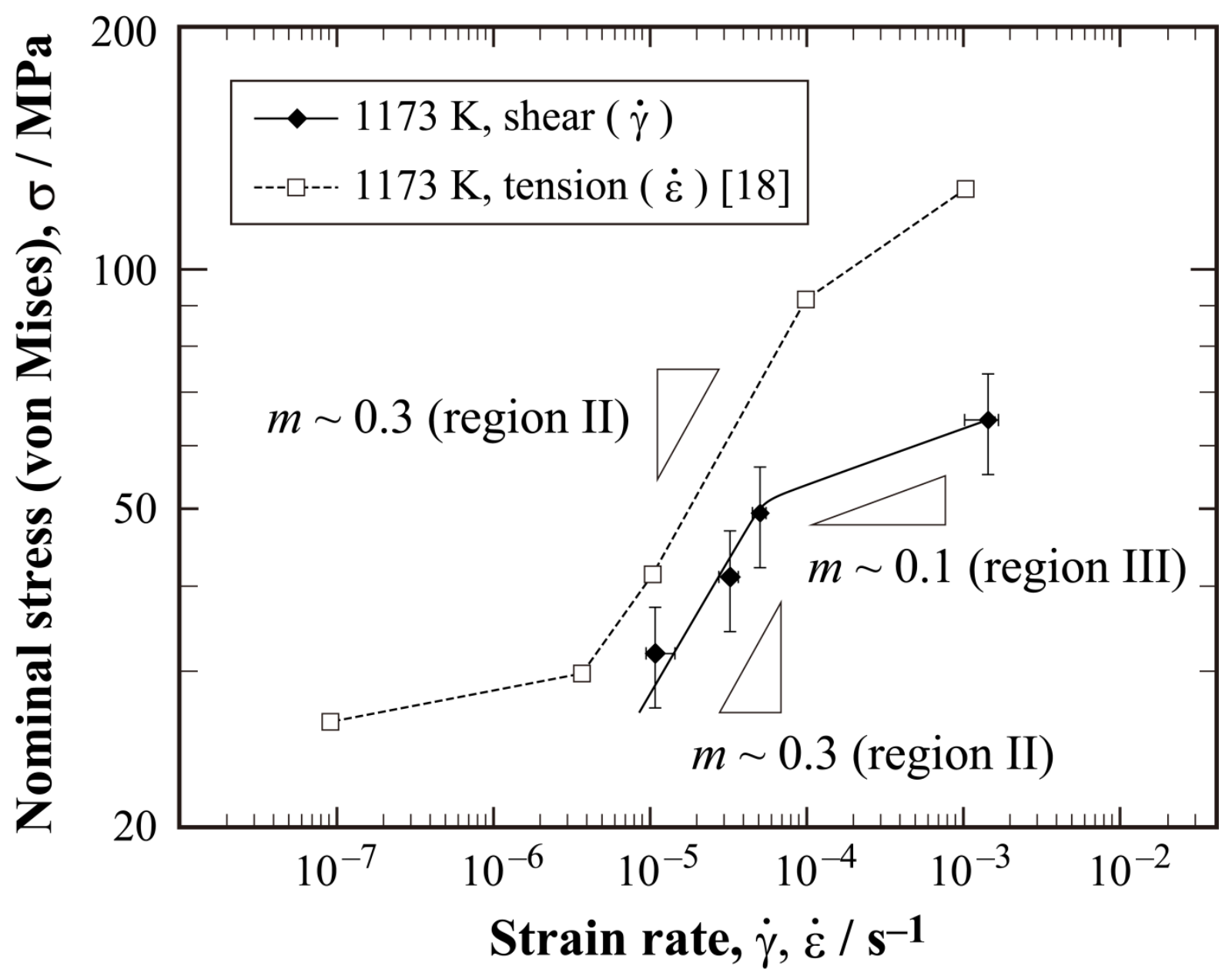

Fig. 5 Nominal stress (von Mises) as a function of average strain at $1173 \mathrm{~K}$. 
(a) Region III (26 N, 12\%)

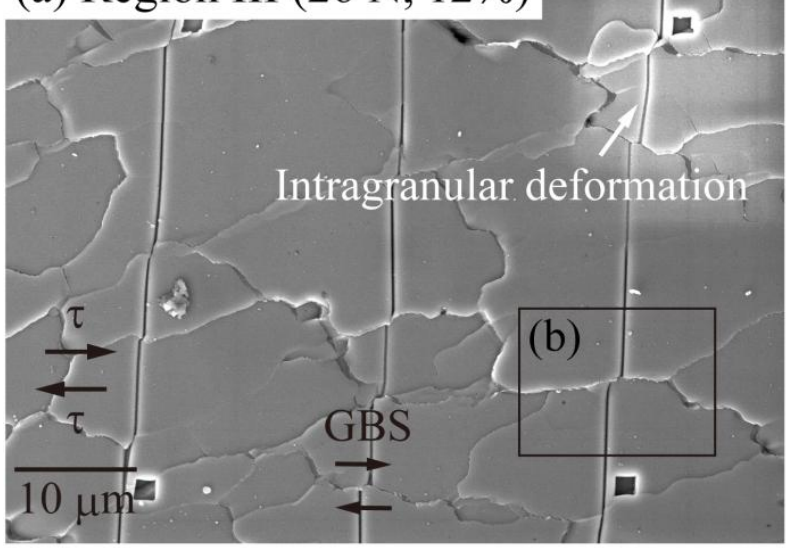

(c) Region II/III (17 N, 10\%)

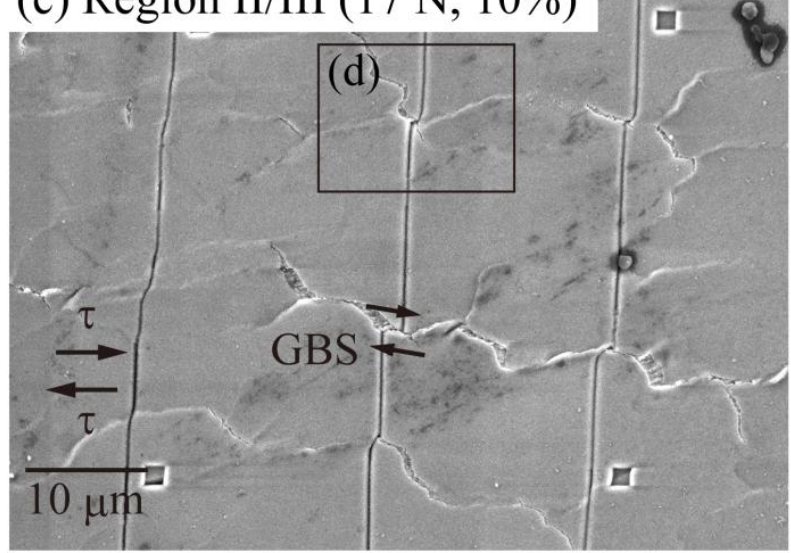

(e) Region II (13 N, 5.1\%)

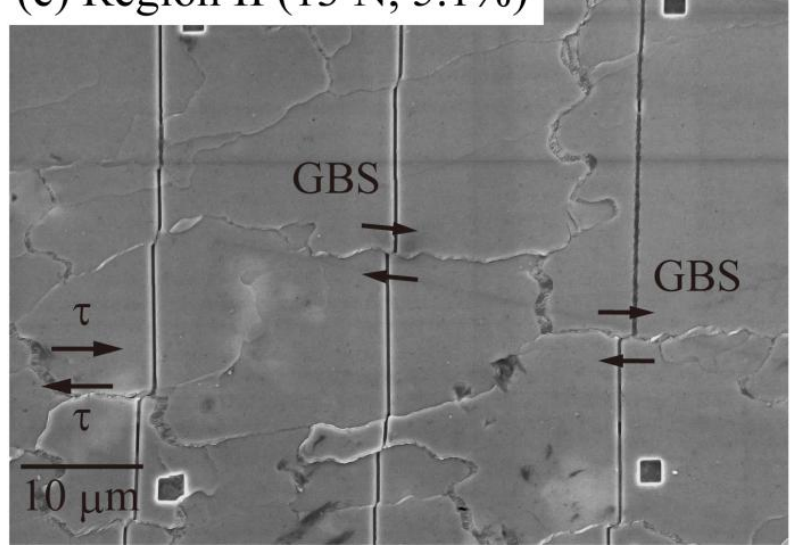

(b) Region III (26 N, 12\%)

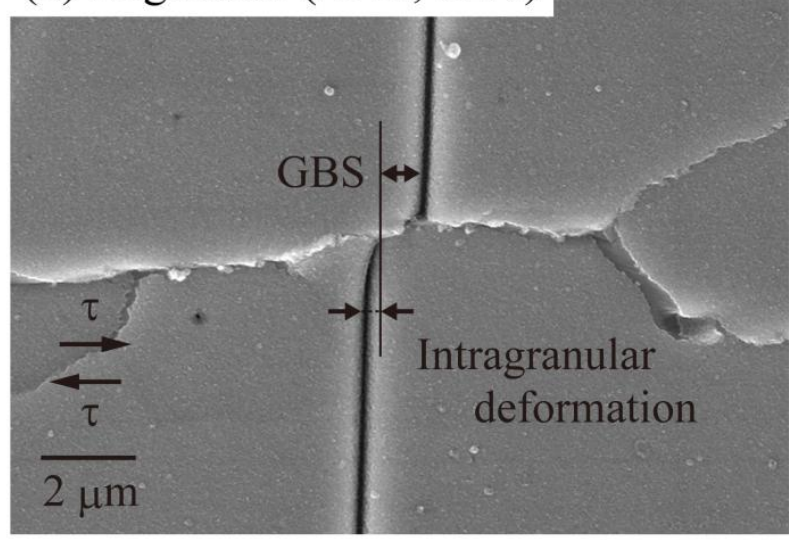

(d) Region II/III (17 N, 10\%)

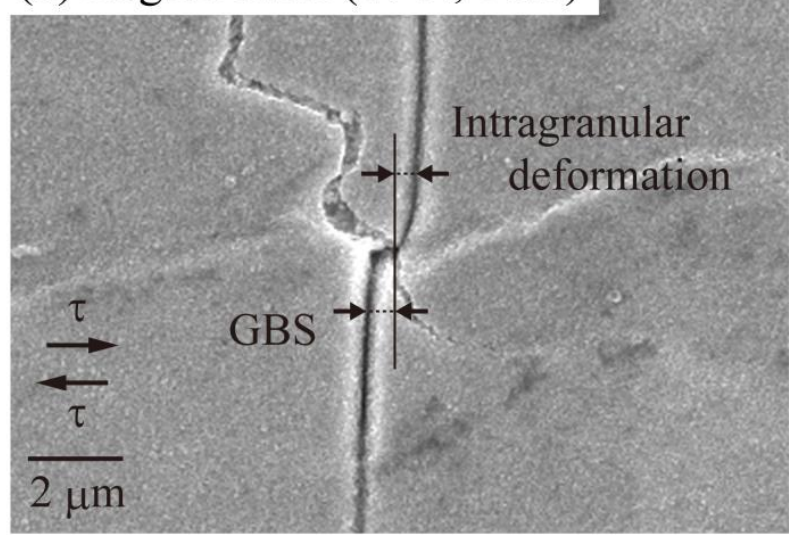

Fig. 6 SEIs after deformations in (a) (b) region III (1173 K, 26 N, 12\%), (c) (d) region II/III (1173 K, $20 \mathrm{~N}, 10 \%)$, and (e) (f) region II (1173 K, $13 \mathrm{~N}, 5 \%)$. (b) and (d) are magnified images of the areas marked by rectangles in (a) and (c), respectively. 

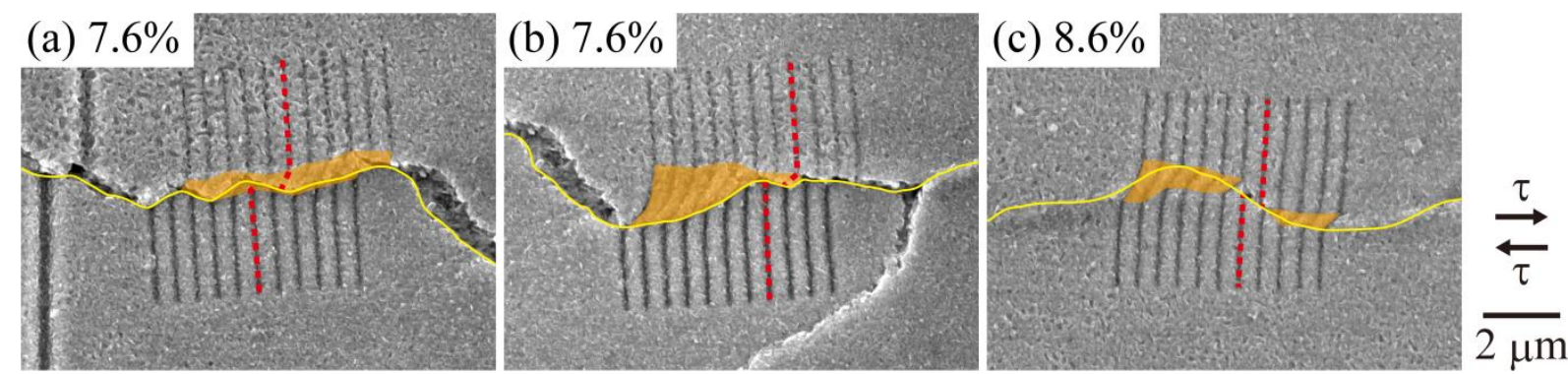

Fig. 7 SEIs after deformation in region II/III $(1173 \mathrm{~K}, 17 \mathrm{~N})$ to mesoscopic shear strains of (a), (b) 7.6\%, and (c) 8.6\%. Yellow lines, orange areas and red dotted lines represent grain boundaries, intragranular deformed areas and original markers, respectively. 

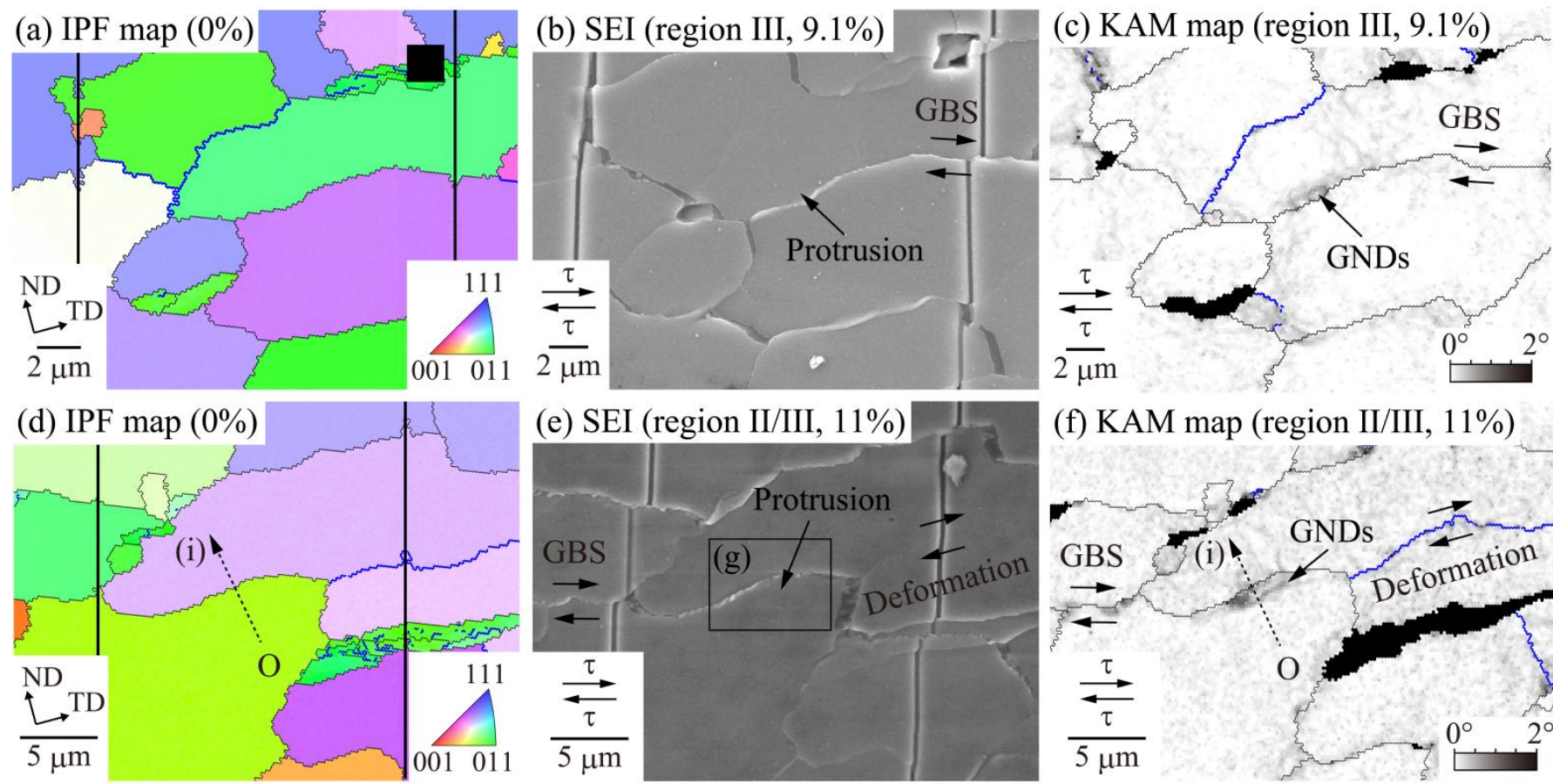

(f) KAM map (region II/III, 11\%)

(g) ECCI (region II/III, 11\%)
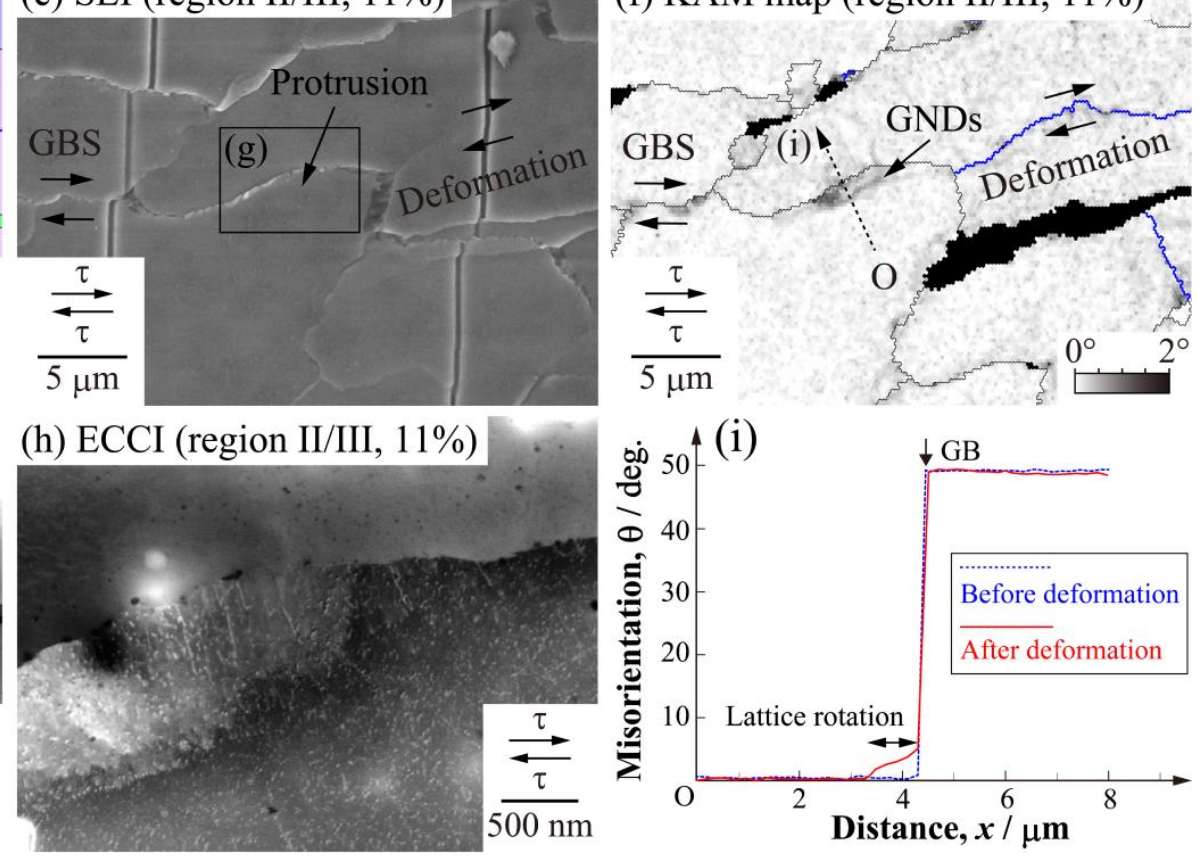

Fig. 8 (a) (d) Initial IPF maps, (b) SEI, and (c) KAM map of region III (1173 K, 26 N,

9.1\%), (e) SEI, (f) KAM map, and (g) (h) ECC images for magnified areas of region II/III

$(1173 \mathrm{~K}, 20 \mathrm{~N}, 11 \%)$, and (i) misorientation profiles along the dashed arrow in (d) and (f). 


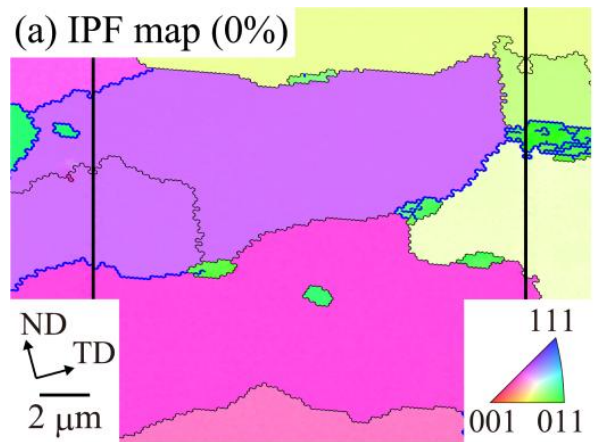

(b) SEI (region III, 9.2\%)

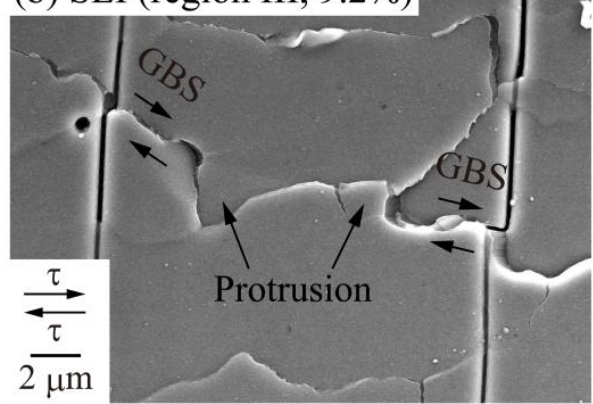

(d) IPF map (0\%)

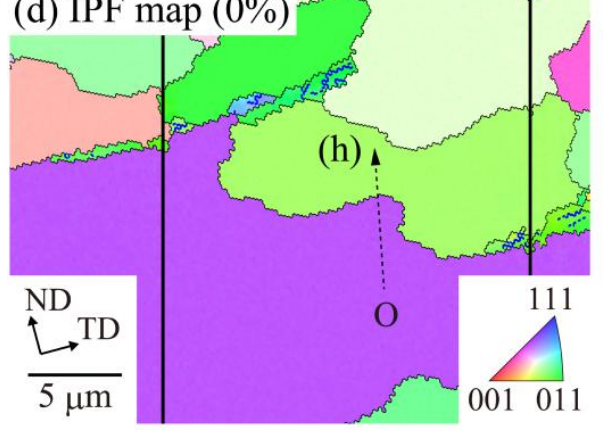

(e) SEI (region II/III, 7.5\%)

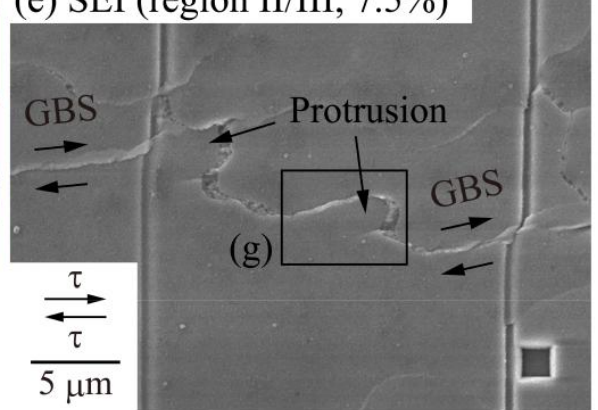

(c) KAM map (region III, 9.2\%)

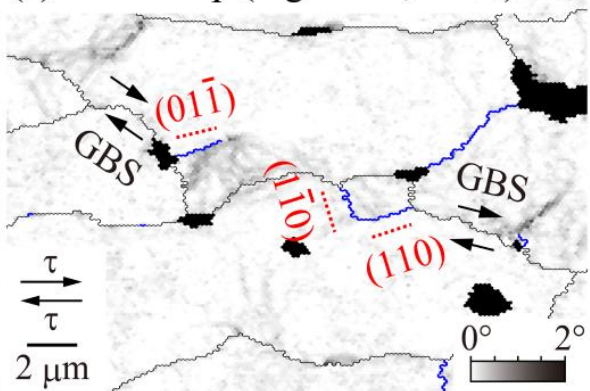

(f) KAM map (region II/III, 7.5\%)

(g) ECCI (region II/III, 7.5\%)
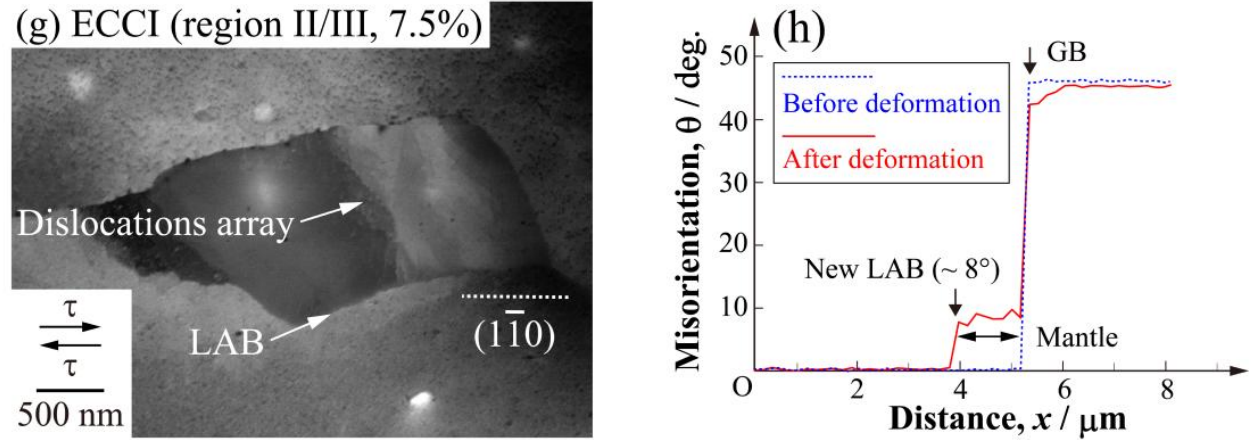

Fig. 9 (a) (d) Initial IPF maps, (b) SEI, and (c) KAM map of region III (1173 K, 26 N, 9.2\%), (e) SEI, (f) KAM map, and (g) ECC images for magnified areas of region II/III (1173 K, 20 N, 7.5\%), and (h) misorientation profiles along the dashed arrow in (d) and (f). 

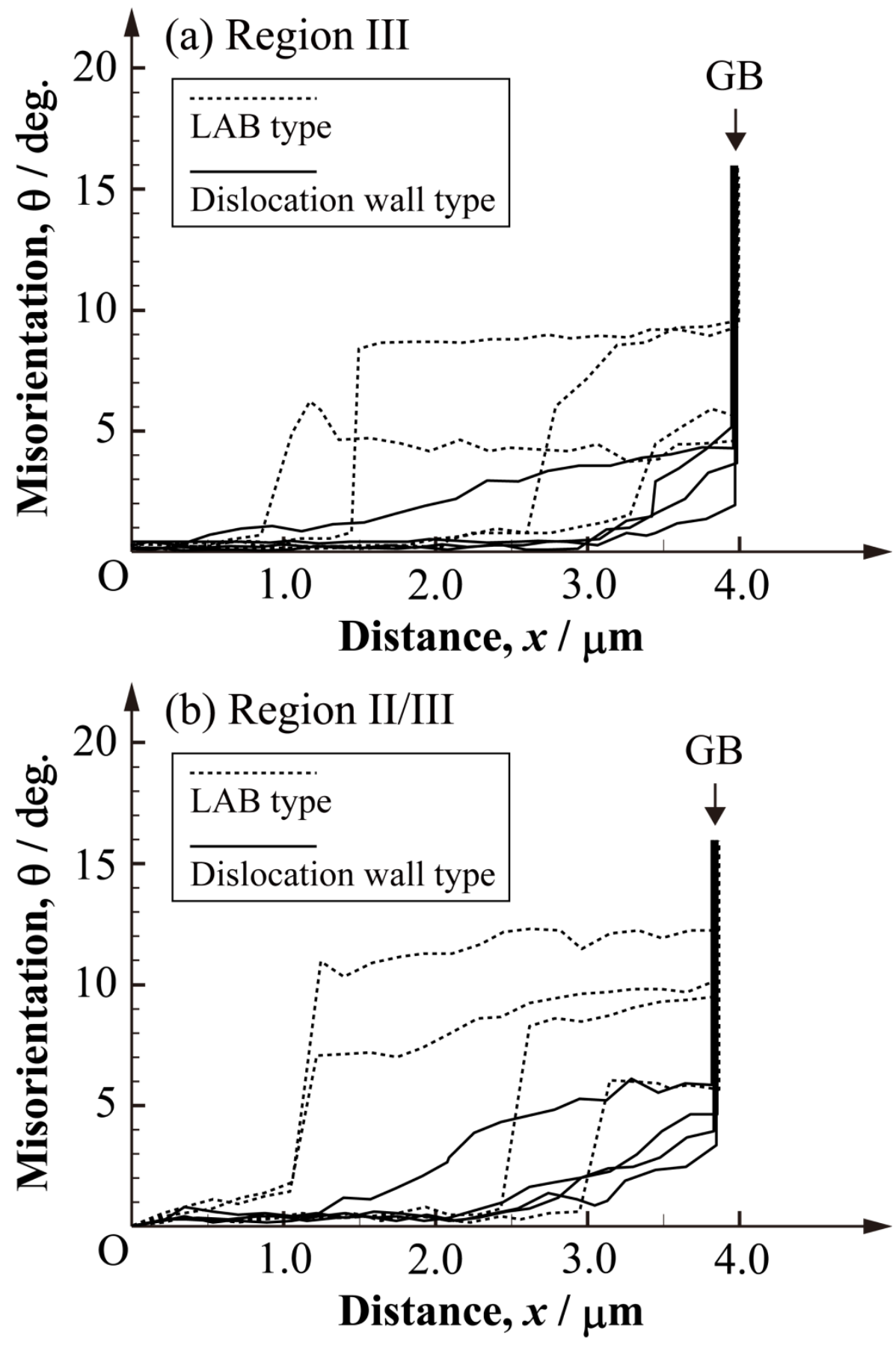

Fig. 10 Misorientation profiles across newly formed substructures around mantle areas randomly chosen after deformations in (a) region III $(1173 \mathrm{~K}, 26 \mathrm{~N})$ and (b) region II/III (1173 K, $20 \mathrm{~N})$. Profiles including LABs (LAB type) and those without an LAB (dislocation wall type) are indicated in dotted and solid lines, respectively. 


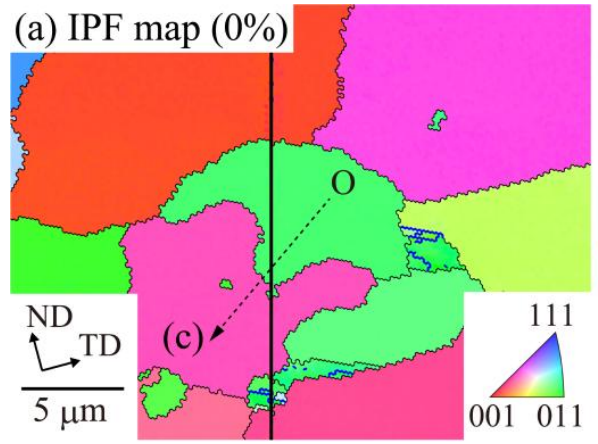

(d) SEI (region III, 18\%)

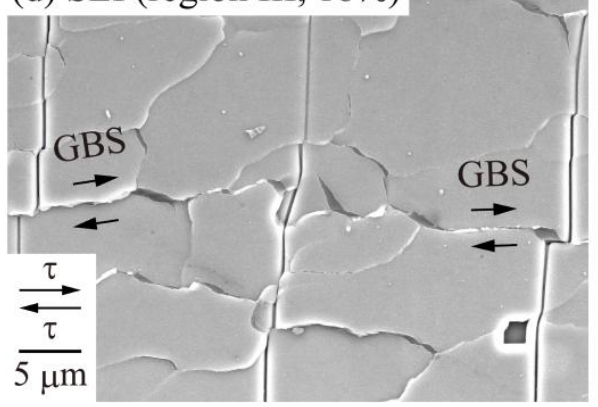

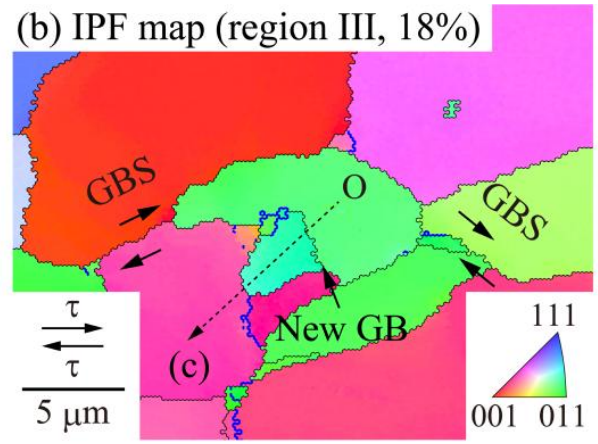

(e) SEI (region III, 18\%)

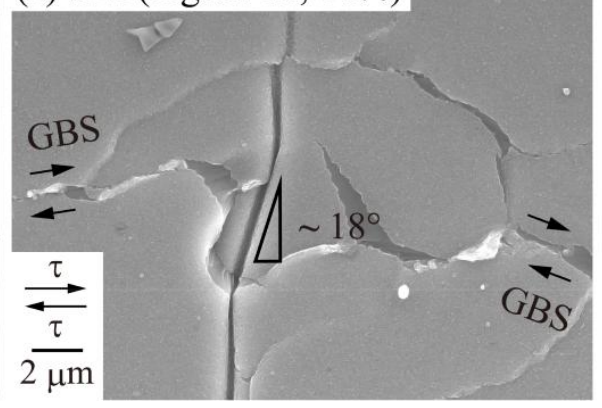

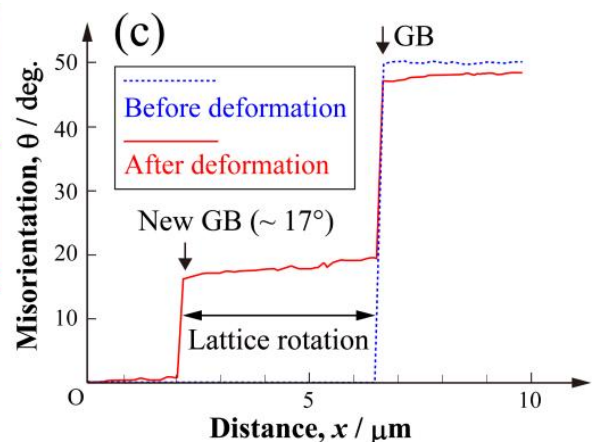

(f) ECCI (region III, 18\%)

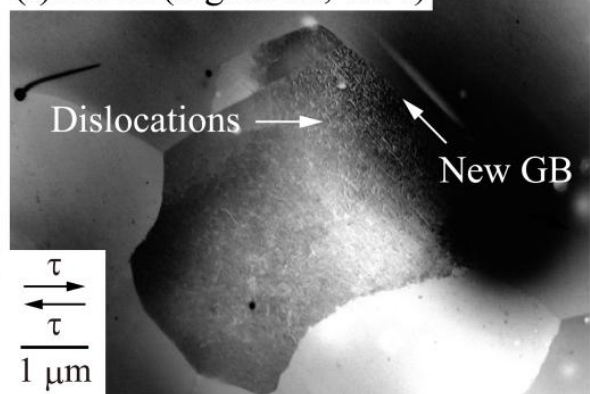

Fig. 11 IPF maps (a) before and (b) after deformation in region III (1173 K, 26 N, 18\%), (c)

misorientation profiles along the dashed arrows in (a) and (b), (d) (e) SEIs of the deformed area, and (f) ECC image of a magnified area. 

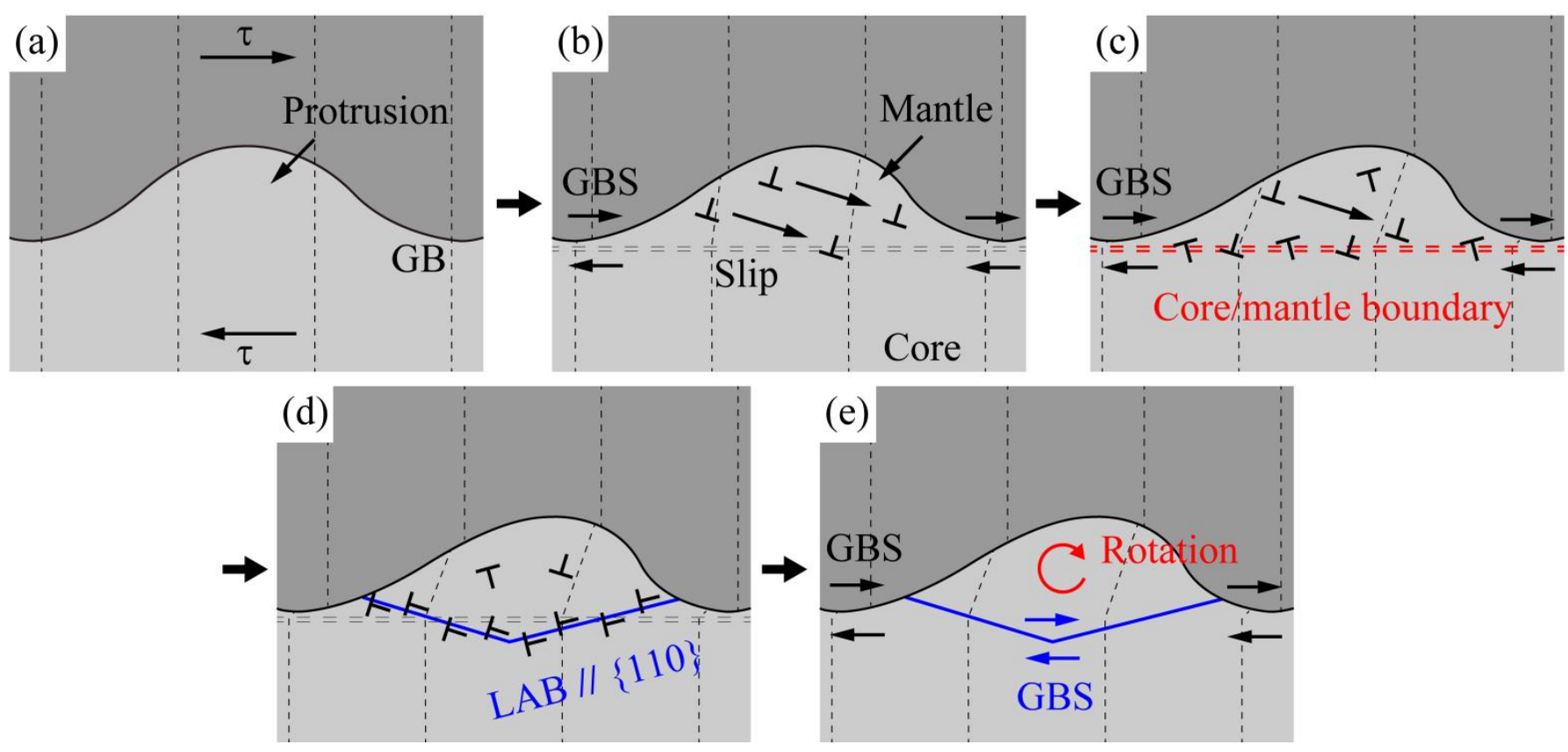

Fig. 12 Proposed mantle dislocation mechanism accommodating GBS: (a) initial state, (b)

dislocation slippage in a mantle accommodating GBS, (c) dislocations trapped at a core/mantle boundary, (d) dynamic recovery of the dislocation structure into an LAB along $\{110\}$ crystallographic planes, and (e) subsequent deformation, such as GBS or grain rotation, compensating for GBS strain. 

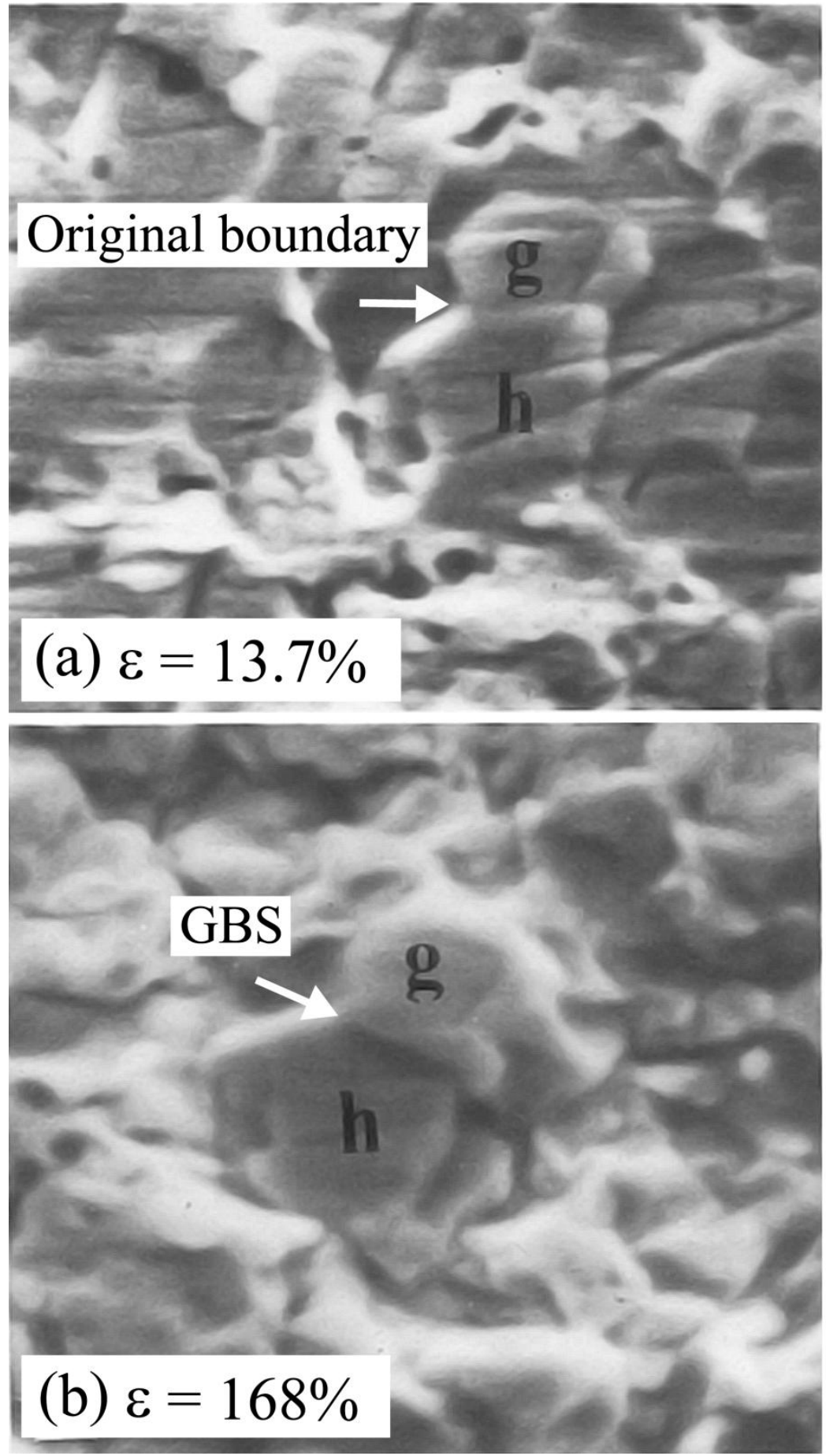

(a)

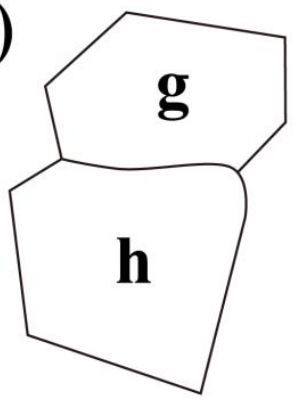

Initial state

(b)

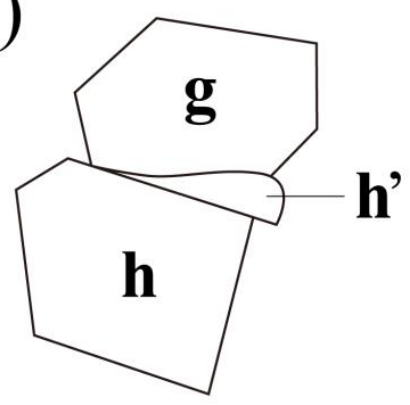

GBS at a new boundary $\mathrm{h} / \mathrm{h}$ '

Fig. 13 SEM photographs during superplastic flow of $\mathrm{Pb}-\mathrm{Sn}$ eutectic at $298 \mathrm{~K}$ at an initial strain rate of $5 \times 10^{-4} \mathrm{~s}^{-1}[24]$ with additional illustrations of deformation by the present authors.

Elongation: (a) $13.7 \%$ and (b) $168 \%$ ( 1976 Springer). 
Table 1 Chemical composition of ODS ferritic steel.

\begin{tabular}{cccccccc}
\hline $\mathrm{Fe}$ & $\mathrm{Cr}$ & $\mathrm{C}$ & $\mathrm{W}$ & $\mathrm{Al}$ & $\mathrm{Zr}$ & $\mathrm{Ti}$ & $\mathrm{Y}_{2} \mathrm{O}_{3}$ \\
\hline Bal. & 15 & 0.03 & 2 & 3.8 & 0.32 & 0.12 & 0.35 \\
\hline
\end{tabular}




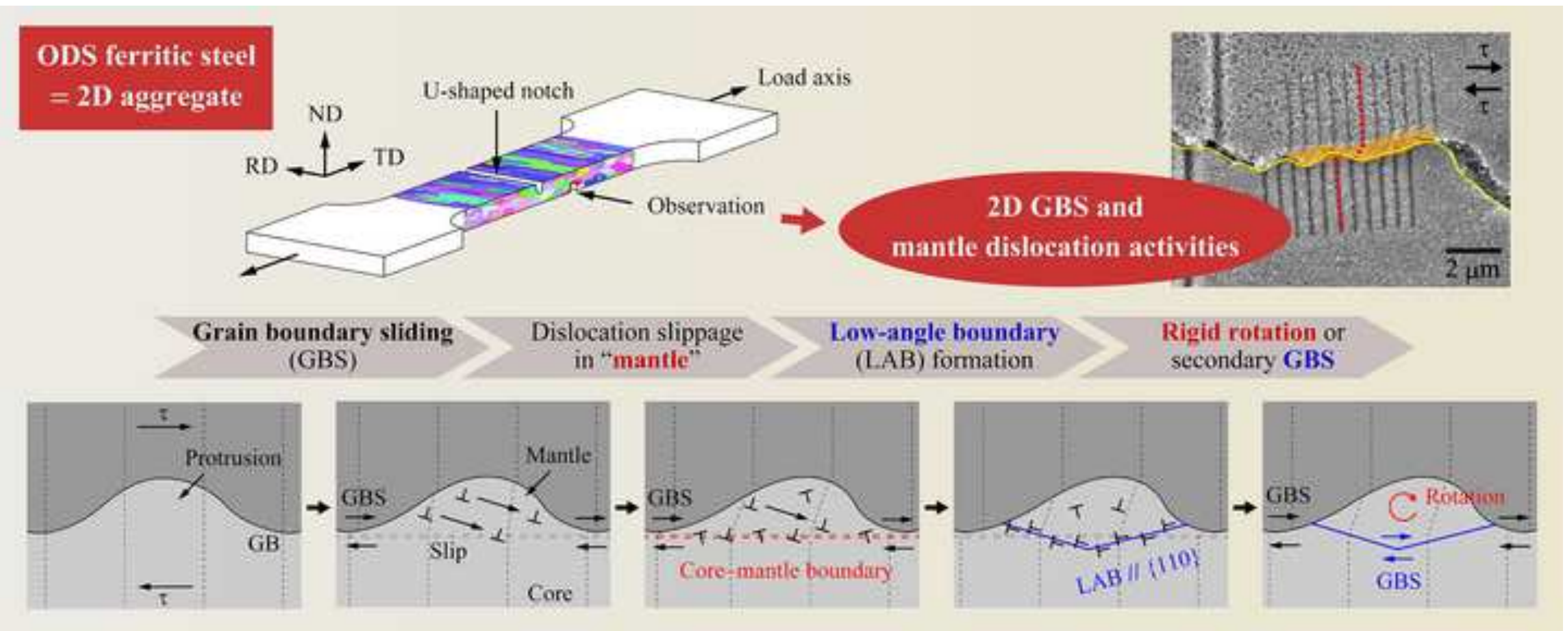

